\title{
Covid-19: Care home deaths in England and Wales double in four weeks
}

\author{
Gareth lacobucci
}

The BMJ

New figures showing a sharp rise in the number of deaths in care homes in England and Wales are "deeply alarming" and demonstrate the need for better surveillance and protection for residents against covid-19, experts have warned.

Official figures from the Office for National Statistics (ONS) show that the number of deaths in care homes from any cause has doubled in the past month since the first deaths from covid-19 were registered.

In the week ending 10 April (week 15), 4927 deaths from any cause were recorded in care homes in England and Wales-a 99\% increase on the 2471 deaths recorded in the week ending 13 March (week 11). In the same one month period the figures show a $72 \%$ increase in deaths from any cause in hospitals (3603 deaths) and a $51 \%$ rise in deaths from any cause in private homes (1392).

Although only 826 deaths were recorded as related to covid-19 at care homes in week 15 and none in week 11, experts suggested that this did not accurately reflect the number of residents dying from infection with the virus.

\section{"Second front"}

Sarah Deeny, assistant director of data analytics at the Health Foundation think tank, said, "The huge increase in deaths from any cause is likely to be a more accurate indication of the scale of the impact of the pandemic on those living in care homes. Better surveillance of deaths in care homes is clearly urgently needed, in a timely manner."

Niall Dickson, chief executive of the NHS Confederation, which represents health and care providers, said, "The sharp rise in care home deaths is deeply alarming: this could be the second front in the battle against covid-19, and we need to do everything we can to support the care sector with the right PPE [personal protective equipment], training and support in infection control, and adequate funding."

The latest ONS data show a spike in overall deaths in England and Wales, as 18516 were registered in week 15 . When based on the five year average this represents 7996 excess deaths, the highest weekly total for over 20 years.

Covid-19 was mentioned in 6213 of the deaths in week 15 (34\%), representing the majority $(78 \%)$ of the excess. In week 14 covid-19 was mentioned in 3475 deaths (21\%).

\section{Indirectly related deaths}

Martin Hibberd, professor of emerging infectious disease at the London School of Hygiene and Tropical Medicine, said, "With limited testing being carried out, it may be that all of the 7996 excess deaths were directly due to covid-19, but it is also likely that at least some of these were indirectly involved, such as through inability to access typical medical care for other conditions because of covid-19 activities."

From next week (28 April) the ONS will publish numbers of care home deaths involving covid-19 based on reporting from care home operators to the regulator, the Care Quality Commission, as the ONS seeks to gain a better understanding of trends.

At a Science Media Centre briefing on 21 April, Carl Heneghan, director of the Centre for Evidence Based Medicine at the University of Oxford, said that the ONS's numbers were consistent with the peak in deaths from the current wave of covid-19 occurring on 8 April.

But he added, "The slight caveat is that once coronavirus is seeded into nursing homes it could create a considerable problem, because the lag between the death and when they are infected will not be clear in the elderly, and the way this infection seems to work is that it can last in the body for 7-14 days.

"So, we might see the deaths in hospitals [from covid-19] come down, but this proportion [dying from covid-19] in nursing homes could have a considerable lag going forward." 